\title{
Regional Myocardial Perfusion Disturbance in Experimental Chronic Chagas Cardiomyopathy
}

\author{
Luciano Fonseca Lemos de Oliveira ${ }^{1}$, James T. Thackeray ${ }^{2}$, José Antônio Marin Neto ${ }^{1}$, Minna Moreira Dias Romano ${ }^{1}$, \\ Eduardo Elias Vieira de Carvalho ${ }^{1}$, Jorge Mejia ${ }^{3}$, Denise Mayumi Tanaka ${ }^{1}$, Grace Kelly da Silva ${ }^{1}$, Douglas Reis Abdalla ${ }^{4}$, \\ Carlos Malamut ${ }^{5}$, Frank M. Bengel ${ }^{2}$, Maria de Lourdes Higuchi ${ }^{6}$, André Schmidt ${ }^{1}$, Edécio Cunha-Neto ${ }^{6}$, \\ and Marcus Vinicius Simões ${ }^{1}$
}

${ }^{I}$ Medical School of Ribeirão Preto, University of São Paulo, Ribeirão Preto, Brazil; ${ }^{2}$ Department of Nuclear Medicine, Hannover Medical School, Hannover, Germany; ${ }^{3}$ Hospital Israelita Albert Einstein, São Paulo, Brazil; ${ }^{4}$ Health Department, Faculty of Human Talent, Uberaba, Brazil; ${ }^{5}$ Nuclear Technology Development Center (CDTN), National Commission of Nuclear Energy (CNEN), Belo Horizonte, Brazil; and ${ }^{6}$ Heart Institute (InCor), Medical School of São Paulo, University of São Paulo, São Paulo, Brazil

\begin{abstract}
Altered myocardial perfusion is a common finding in chronic Chagas cardiomyopathy (CCC), but its underlying histologic changes have not been elucidated. We investigated the occurrence of myocardial perfusion defects (MPDs) and the correlated regional changes to histology in an experimental model of CCC in hamsters. Methods: Female Syrian hamsters $(n=34)$ were infected with $3.5 \times 10^{4}$ to $10^{5}$ trypomastigote forms of Trypanosoma cruzi, $Y$ strain, and 6-10 mo afterward underwent in vivo imaging including resting ${ }^{99 \mathrm{~m} T c-s e s t a-}$ mibi SPECT, segmental and global left ventricular function assessment using 2-dimensional echocardiography, and ${ }^{18} \mathrm{~F}-\mathrm{FDG}$ PET for evaluation of myocardial viability. Histologic analysis included quantification of fibrosis, inflammatory infiltration, and the diameter and density of myocardial microcirculation. Results: MPDs were present in $17(50 \%)$ of the infected animals. Histologic analysis revealed no transmural scar in segments with an MPD, and normal or mildly reduced ${ }^{18} \mathrm{~F}-\mathrm{FDG}$ uptake, indicating viable myocardium. Infected animals with an MPD, in comparison to infected animals without an MPD and control animals, showed a lower left ventricular ejection fraction $(P=0.012)$, a higher wall motion score index $(P=0.004)$, and a higher extent of inflammatory infiltration $(P=0.018)$ but a similar extent of fibrosis $(P=0.15)$ and similar microvascular diameter and density $(P>0.05)$. Segments with an MPD $(n=65)$, as compared with normally perfused regions in the same animal $(n=156)$, showed a higher wall motion score index $(P=0.005)$ but a similar extent of inflammatory infiltration, a similar extent of fibrosis, and a similar microvascular diameter and density. Conclusion: Resting MPDs are frequent in experimental CCC and are associated with myocardial inflammation but do not designate scar tissue, corresponding to regions with metabolically viable myocardium.
\end{abstract}

Key Words: chronic Chagas cardiomyopathy; myocardial perfusion; myocardial inflammation

J Nucl Med 2018; 59:1430-1436

DOI: 10.2967/jnumed.117.205450

\footnotetext{
Received Nov. 17, 2017; revision accepted Mar. 22, 2018.

For correspondence or reprints contact: Marcus Vinicius Simões, Medical School of Ribeirão Preto, University of São Paulo, 3900 Bandeirantes Ave., Ribeirão Preto, São Paulo 14048-900, Brazil.

E-mail: msimoes@fmrp.usp.br

Published online Apr. 26, 2018.

COPYRIGHT (C 2018 by the Society of Nuclear Medicine and Molecular Imaging.
}

C hagas disease is a serious public health problem in endemic and nonendemic countries, with 6-7 million cases throughout the world. The disease imparts high morbidity and mortality (1), and because of migratory currents and globalization, is becoming more common worldwide $(2,3)$.

Although most affected individuals never show clinical symptoms, approximately $30 \%$ progress to chronic clinical disease, of which heart involvement is the most severe condition, frequently manifesting as dilated cardiomyopathy with heart failure syndrome, cardiac arrhythmia, thromboembolic events, and sudden death (4). Additionally, 30\%-40\% of patients have anginalike chest pain associated with ischemic electrocardiographic changes (5), segmental wall motion impairment (6), and reversible myocardial perfusion defects (MPDs) (7), which may lead to ischemic cardiomyopathy (8). However, coronary angiograms reveal normal subepicardial coronary arteries $(9,10)$, suggesting coronary microvascular dysfunction $(7,11,12)$.

This hypothesis of coronary microvascular dysfunction in chronic Chagas cardiomyopathy (CCC) is supported by experimental histologic and autopsy evidence showing structural changes in coronary microvessels, such as intima proliferation and luminal obstruction (13), formation of platelet plugs (14), arteriolar spasms (15), and vascular rarefaction (16). Despite relevance to clinical manifestation and pathophysiology, a topographic correlation between changes in perfusion and underlying histopathologic alterations in CCC has not been explored in vivo.

We hypothesized that regional myocardial perfusion abnormalities would align topographically with regions of systolic dysfunction. Furthermore, we assessed inflammation, fibrosis, and microcirculation by histopathology to delineate the contribution of these alterations to perfusion.

\section{MATERIALS AND METHODS}

\section{Experimental Animals}

Female Syrian hamsters (12 wk old) were maintained in temperature-controlled accommodations with free access to food and water under a 12-h light/dark cycle. Animals $(n=60)$ were inoculated intraperitoneally with $3.5 \times 10^{4}$ to $1 \times 10^{5}$ blood trypomastigote forms of Trypanosoma cruzi, Y strain. Control animals $(n=8)$ received an equal volume of saline. All studies were approved by the institutional Research Committee on Animal Experimentation of our institution 
(approval 034/2011) and conducted in accordance with the recommendations of the Brazilian System of the National Council for the Control of Animal Experimentation.

\section{Study Design}

The total mortality of the infected animals was $43 \%$ throughout the acute $(33 \%, n=20)$ and chronic $(10 \%, n=6)$ phases, and only 1 control animal died as a consequence of anesthesia during the imaging studies. The 34 infected surviving animals underwent imaging including echocardiography and myocardial perfusion scintigraphy at 6 mo $(n=28)$ and $10 \mathrm{mo}(n=6)$ after infection. All surviving control animals $(n=7)$ were investigated at 6 mo. Selection of these time points to evaluate the chronic phase of the disease with variable left ventricular (LV) dysfunction was based on the literature (17). After imaging, the animals were euthanized, and blood samples were collected to confirm chronic Chagas infection (18). Cardiac tissue samples were collected for histopathologic examination.

An additional independent experiment was conducted to evaluate myocardial viability using in vivo imaging. For this experiment, an additional group of 10-mo T. cruzi-infected animals $(n=4)$ and controls $(n=2)$ underwent ${ }^{18} \mathrm{~F}$-FDG PET for evaluation of myocardial viability in regions with severe resting MPDs.

\section{Echocardiogram}

Myocardial systolic function was assessed using high-resolution 2dimensional echocardiography (HD11XE; Philips) with a $15-\mathrm{MHz}$ linear transducer (19). LV ejection fraction (LVEF) was determined by the Teichholz method, and regional systolic function was determined by average wall motion score index (WMSi)

\section{Myocardial Perfusion Scintigraphy}

Myocardial perfusion was determined using a validated high-resolution SPECT adaptation of a clinical $\gamma$-camera with a single $1.5-\mathrm{mm}$ pinhole collimator, an aperture angle of $150^{\circ}$, and shielding of photons with energy up to $150 \mathrm{keV} \mathrm{(20).}$

The animals were anesthetized with sodium pentobarbital $(50 \mathrm{mg} / \mathrm{kg})$ and injected via the internal jugular vein with $555 \mathrm{MBq}$ of ${ }^{99 \mathrm{~m}} \mathrm{Tc}-$ sestamibi. After $90 \mathrm{~min}$ of uptake, the animals were reanesthetized and imaged over $360^{\circ}$ with 40 projections and $30 \mathrm{~s}$ per view. The images were reconstructed to a $128 \times 128$ matrix $(0.54-\mathrm{mm}$ pixel size).

Radiotracer accumulation in the myocardium was analyzed semiquantitatively using polar maps (21). Hypoperfusion was defined as less than 50\% uptake compared with the maximum. MPD extent was the total defect area relative to total LV surface (\%). A 13-segment model was used for regional analysis compared with echocardiography (Fig. 1).

\section{Viability PET Imaging}

${ }^{18}$ F-FDG PET images were obtained using a dedicated smallanimal camera (LabPET 4 Solo; GE Healthcare).

Hamsters received $70 \mathrm{MBq}$ of ${ }^{18} \mathrm{~F}-\mathrm{FDG}$ via the sublingual vein $20 \mathrm{~min}$ after isoflurane anesthesia (3\%-4\% in 100\% oxygen) to increase myocardial ${ }^{18} \mathrm{~F}-\mathrm{FDG}$ uptake, as previously described (22). ${ }^{18} \mathrm{~F}$-FDG uptake for $60 \mathrm{~min}$ in conscious animals was followed by a second isoflurane induction and a 15-min static PET scan at 60-75 min after injection.
The images were reconstructed to a $128 \times 128 \times 159$ matrix $(0.78 \times$ $0.78 \times 0.80 \mathrm{~mm}$ ) using 10-iteration 3-dimensional maximum-likelihood expectation maximization with scanner-applied scatter correction using LabPET, version 1.12.0 (Gamma Medica-Ideas). No attenuation correction was applied.

Polar maps were analyzed to define regional viability. Segments with less than $50 \%$ of the maximum ${ }^{18} \mathrm{~F}$-FDG signal were considered nonviable.

\section{Histopathology}

After euthanasia, the hearts were excised, fixed, and embedded in paraffin. Sections (5 $\mu \mathrm{m}$ thick) were stained with hematoxylin-eosin or picrosirius red for quantitation of inflammation and fibrosis, respectively. Basal, mid-ventricular, and apical sections were analyzed to correlate histologic segments with in vivo images. Digital microscopy images $\left(1.5-2 \mathrm{~mm}^{2}\right)$ were analyzed using Aperio ImageScope viewing software (Leica Biosystems). Inflammation was quantified by the number of mononucleated cells per field $\left(\right.$ cells $\left./ \mathrm{mm}^{2}\right)$. Picrosirius red staining defined the interstitial and perivascular fibrosis as a percentage of the total area.

\section{Immunohistochemistry}

To determine the microcirculation vessel density and diameter, $\alpha$-actin antibody was used to label vascular smooth muscle as previously described (23). Microscopic analysis of the $\alpha$-actin immunohistochemistry images identified and enumerated fine-caliber vessels with identifiable walls, a visible lumen, and intramyocardial localization as intramural microvessels.

\section{Statistical Analysis}

Continuous variables are reported as mean $\pm \mathrm{SD}$, and nominal variables are reported as absolute $(n)$ and relative (\%) frequency. Statistical analyses were performed using InStat software, version 3.05 (Graph$\mathrm{Pad}$ ). The Kolmogorov-Smirnov test determined the normality of data distribution. Differences between the means of 2 groups were analyzed 
by the Student $t$ test for variables of normal distribution and by the Mann-Whitney test for variables of nonnormal distribution. Simultaneous comparative analysis of 3 or more groups was performed by ANOVA with the Tukey posttest for variables of normal distribution and by the Kruskal-Wallis test with the Dunn posttest for variables of nonnormal distribution. The Fisher exact test was used to determine the heterogeneity of frequency distribution. Correlations were determined by the Pearson test for variables of normal distribution and by the Spearman test for variables of nonnormal distribution. The level of significance was set at $5 \%$ in all analyses $(P<0.05)$.

\section{RESULTS}

\section{Systolic Function}

Contractile dysfunction was defined as $73 \%$ on the basis of the mean and SD in healthy control hamsters $(82.0 \% \pm 4.6 \%)$. WMSi did not change in the control group. Eighteen of 34 infected hamsters displayed abnormal LV function, with only segmental LV dysfunction in 8 animals (44\%) and global systolic dysfunction $(\mathrm{LVEF}<73 \%)$ in 10 animals $(56 \%)$.

All animals with a reduced LVEF exhibited a diffuse reduction in wall motion. An apical wall motion abnormality was present in all animals, comprising akinesis $(8 / 18,44 \%)$ and hypokinesis $(10 /$ $18,56 \%)$. Hypokinetic wall motion was also observed in the inferior segment (8/18) and posterolateral segment (10/18).

\section{Myocardial Perfusion Assessment}

Control animals exhibited homogeneous perfusion, though mild MPDs (1\%-5\% of the LV apical surface) were observed. Accordingly, the threshold for MPDs in infected animals was limited to $5 \%$ of the LV surface.

Significant MPDs were present in $17(50 \%)$ of the infected animals, including 12 animals at 6 mo (43\%) and 5 animals at $10 \mathrm{mo}(83 \%)$ after infection, with an MPD area of $17.1 \% \pm 13.1 \%$ of the LV surface (range, 6.4\%-59\%). The mean MPD area was larger in the 10 -mo animals $(27.7 \% \pm 19.0 \%)$ than in the 6-mo animals $(12.7 \% \pm 6.8 \%, P=0.01)$.

The apical region displayed an MPD in all cases. MPDs were frequently detected in mid-anterolateral $(71 \%)$ and mid-anterior (47\%) segments (Fig. 2A) and were diffuse and less frequent in other segments (Fig. 2B).

\section{Perfusion Changes Corresponding to Systolic LV \\ Dysfunction}

Infected animals with MPD exhibited lower LVEF $(65.2 \% \pm 19.2 \%)$ compared with infected animals without MPD $(79.1 \% \pm 12 \%$,

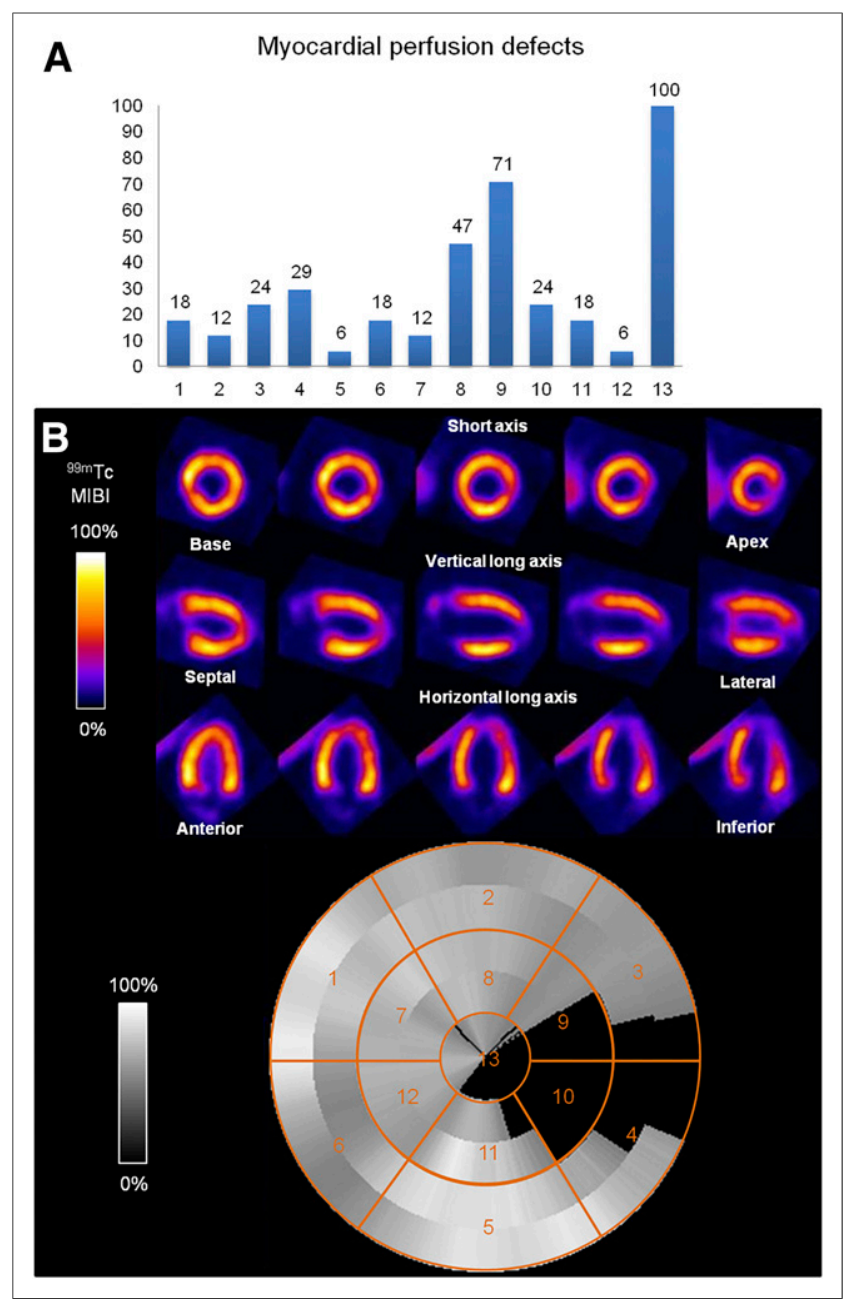

FIGURE 2. (A) Bar graph illustrating relative incidence of MDPs according to topographic distribution. $y$-axis is percentage of MDP; $x$-axis is segment number in 13-segment model. (B) ${ }^{99 m} T c$-sestamibi SPECT images of myocardial perfusion 10 mo after infection, with corresponding polar map. Perfusion defect area corresponds to $24 \%$ of surface LV area, involving apical segments and posterolateral and anterolateral walls.

$P<0.05)$ and control animals $(81.9 \% \pm 4.6 \%, P<0.05), P=$ 0.012 (variance analysis [ANOVA]). The WMSi for animals with altered perfusion was $38 \%$ higher than controls $(P=0.004)$ (Table 1$)$.

TABLE 1

Ventricular Function Data and Histologic Changes

\begin{tabular}{|c|c|c|c|c|}
\hline Parameter & Control $(n=7)$ & CCC with normal perfusion $(n=17)$ & CCC with MPD $(n=17)$ & $P$ \\
\hline LVEF (\%) & $81.9 \pm 4.6$ & $79.1 \pm 12$ & $65.2 \pm 19.2^{*}$ & 0.012 \\
\hline WMSi & $1.0 \pm 0$ & $1.1 \pm 0.3$ & $1.4 \pm 0.5^{\dagger}$ & 0.004 \\
\hline Inflammation $\left(\mathrm{n} / \mathrm{mm}^{2}\right)$ & $113.2 \pm 38.8$ & $108.2 \pm 53.6$ & $176.2 \pm 86.8^{\ddagger}$ & 0.018 \\
\hline Fibrosis (\%) & $4.5 \pm 1.8$ & $7.0 \pm 3.1$ & $8.0 \pm 4.1$ & 0.15 \\
\hline \multicolumn{5}{|c|}{$\begin{array}{l}{ }^{\star} P<0.05 \text { vs. control and } \mathrm{CCC} \text { animals with normal myocardial perfusion. } \\
{ }^{\dagger} P<0.05 \text { vs. control animals. } \\
{ }^{\ddagger} P<0.05 \text { vs. } \mathrm{CCC} \text { animals with normal myocardial perfusion. } \\
\text { Data are mean } \pm \mathrm{SD} \text {. }\end{array}$} \\
\hline
\end{tabular}



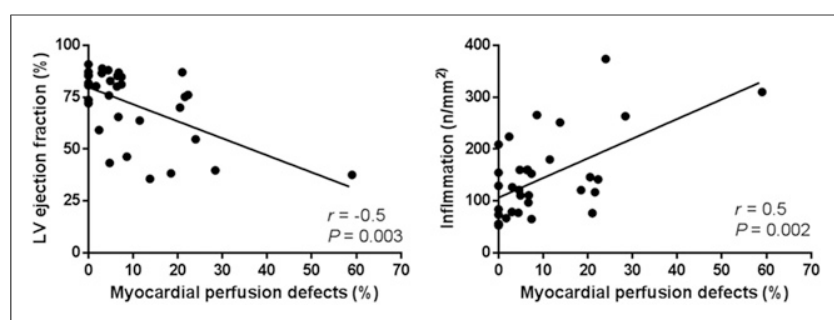

FIGURE 3. Scatterplots illustrating correlation of individual MPD percentages with LVEF and intensity of inflammation.

A significant association was observed between the presence of MPDs and the detection of changes in WMSi in the infected animals. Twelve of 17 animals with MPDs (70\%) showed concurrent wall motion abnormalities, whereas only $6(25 \%)$ of 24 animals with normal perfusion displayed altered wall motion $(P=$ 0.005 , Fisher exact test). The MPD area in infected animals was inversely correlated with LVEF ( $r=-0.502, P=0.003$ ) (Fig. 3).

Segments with MPDs $(n=65)$ displayed a higher segmental motion score $(1.6 \pm 0.7)$ than segments with normal perfusion $(n=156 ; 1.3 \pm 0.5)(P=0.005)$.

MDPs were topographically associated with concomitant WMSi changes: 33 of 65 segments with abnormal perfusion $(50.7 \%)$ exhibited simultaneous WMSi changes (7 akinetic, 25 hypokinetic), whereas only 48 of 156 segments with normal perfusion $(30.7 \%)$ showed concomitant changes in wall motion $(P=0.006$; Fisher exact test).

\section{Histopathologic Analysis}

Qualitative analysis revealed diffuse and multifocal mononuclear cell infiltration with fiber degeneration and variable myocytolytic injury associated with modest interstitial and perivascular fibrosis. No atherosclerotic plaques or platelet plugs were detected in the macro- or microcirculation.

Notably, segments with MPDs did not correspond to regions with significant transmural fibrosis (Fig. 4). However, infected animals with MPDs showed higher inflammation than infected animals with normal perfusion $\left(176.2 \pm 86.8\right.$ and $108.2 \pm 53.6 \mathrm{n} / \mathrm{mm}^{2}$, $P<0.05)$ and a similar extent of fibrosis $(8 \% \pm 4.1 \%$ and $7 \% \pm$ $3.1 \% / \mathrm{mm}^{2}, P=0.15$ ) (Table 1 ).

Regional analysis between segments with and without MPDs in individual animals demonstrated a similar inflammation intensity $\left(196.7 \pm 113.5\right.$ vs. $176.4 \pm 110.4$ cells $\left./ \mathrm{mm}^{2}, P=0.15\right)$ and fibrosis extent $\left(8.8 \% \pm 5.5 \%\right.$ vs. $\left.7.7 \% \pm 5.2 \% / \mathrm{mm}^{2}, P=0.11\right)$. Among infected animals, the MPD area directly correlated with the intensity of inflammation ( $r=0.51, P=0.002$; Fig. 3) but not with the extent of myocardial fibrosis $(r=0.22, P=0.217)$.

\section{Myocardial Viability Assessed by ${ }^{\mathbf{1 8}}$ F-FDG PET}

Control animals showed homogeneous perfusion and myocardial ${ }^{18}$ F-FDG uptake. In the infected group, 3 animals had resting MPDs $(28 \%, 19 \%$, and $8 \%)$. The segments exhibiting MPDs showed only a mild reduction of myocardial ${ }^{18}$ F-FDG uptake (i.e., $>50 \%$ of maximum), indicating a perfusion-metabolism mismatch and viable myocardium with resting hypoperfusion (Fig. 5).

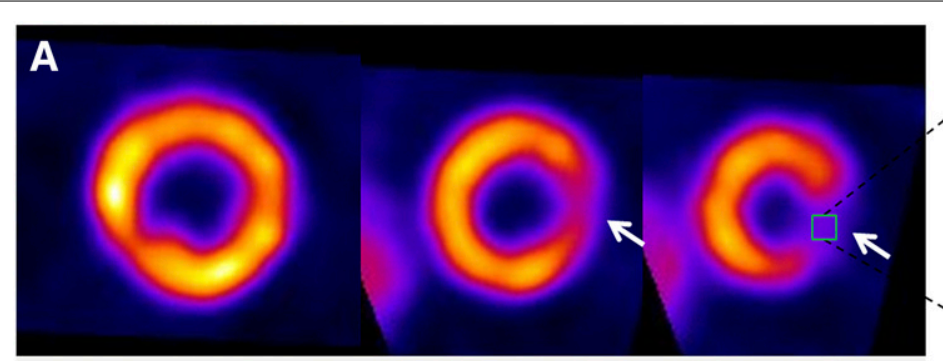

B
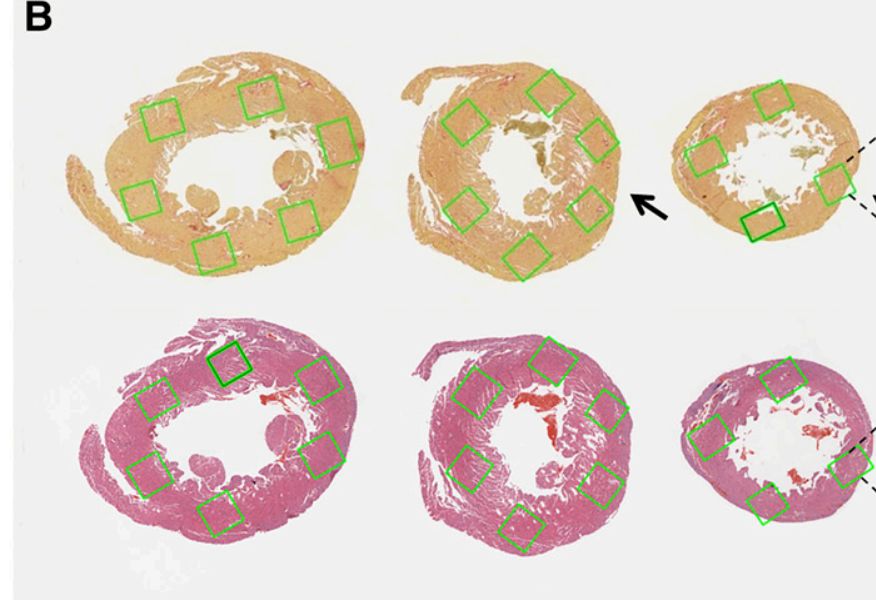
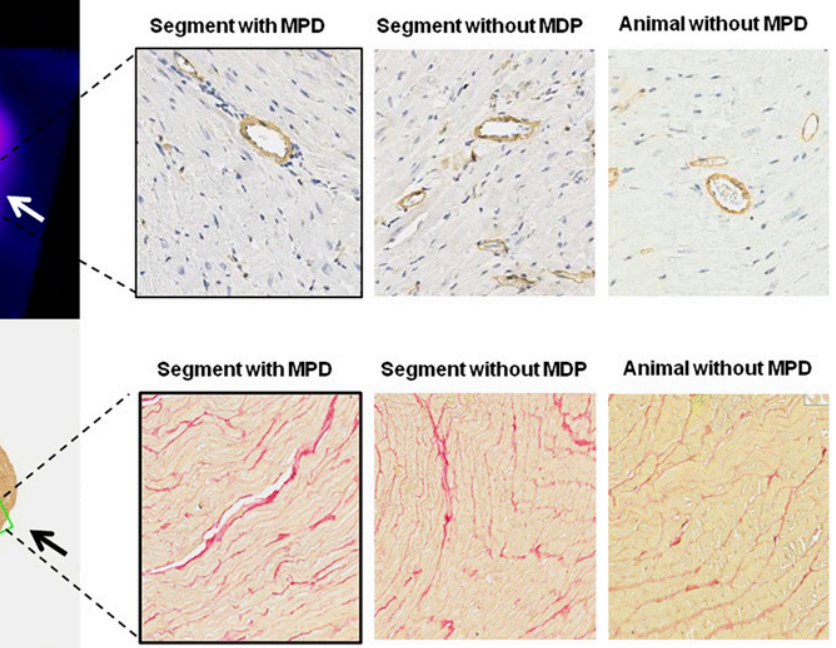

Segment with MPD

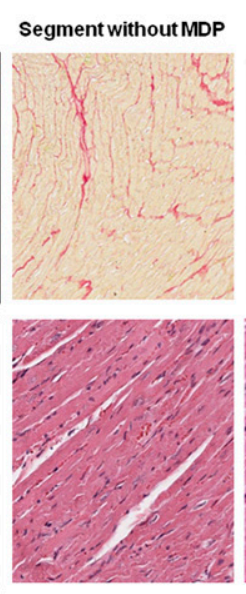

FIGURE 4. (A) At left, SPECT images of myocardial perfusion reveal severe MPD involving mid posterolateral and apical segments (white). At right, correlative immunohistochemistry study shows no differences in diameter or density of microvessels between segments or animals with and without MPD. (B) Picrosirius red-stained histologic sections at top left show no transmural fibrosis in the same regions as correlated with in vivo images (black arrows) presenting MPD; histologic study at top right showed no differences in extent of fibrosis between segments or animals with and without MPD. Hematoxylin- and eosin-stained histologic sections at bottom show mild, diffuse mononuclear infiltrating cells in animals with MPD, however, no differences were observed between segments with and without MPD in the same animal. 


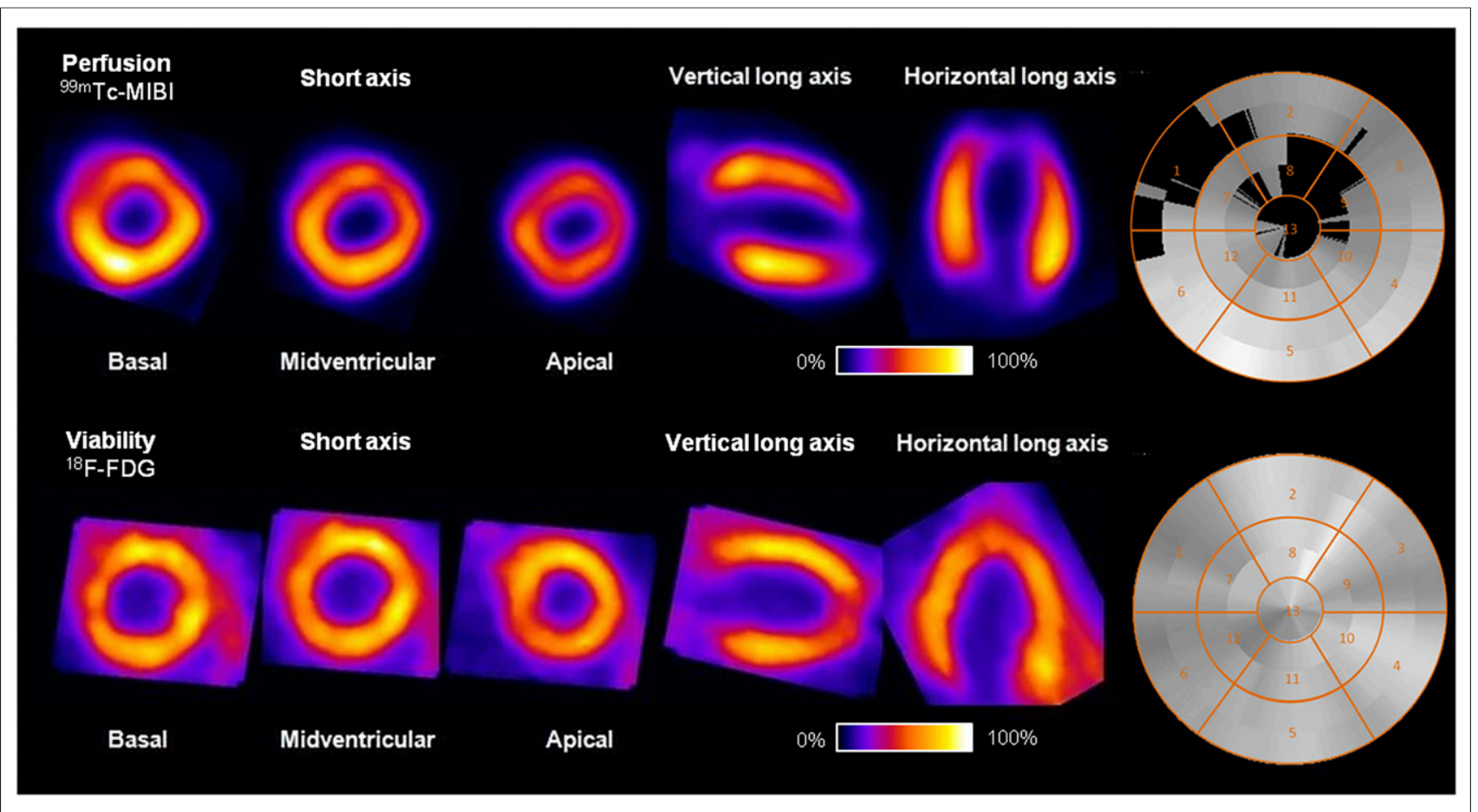

FIGURE 5. At top, high-resolution ${ }^{99 m T C-s e s t a m i b i ~ S P E C T ~ i m a g e s ~ s h o w ~ s e v e r e ~ M D P ~}(<50 \%$ uptake) involving $28 \%$ of LV surface in basal septoanterior, mid-anterior, mid-anterolateral, and apical segments. At bottom, ${ }^{18} \mathrm{~F}-\mathrm{FDG}$ PET images of corresponding tomographic sections show preserved radiotracer uptake, demonstrating myocardial viability in area of MDP (mismatch).

\section{Coronary Microvasculature}

No significant differences between $\mathrm{CCC}$ and control animals were observed in the diameter $(7.8 \pm 0.8$ vs. $7.5 \pm 0.7 \mu \mathrm{m}, P=0.4)$ or density $\left(22.3 \pm 2.4\right.$ vs. $26 \pm 10.1$ vessels $\left./ \mathrm{mm}^{2}\right)$ of the vessels of the coronary microcirculation $(P=0.08)$. Likewise, infected animals with and without MPDs showed similar vessel diameters $(7.7 \pm$ 0.7 vs. $7.9 \pm 0.9 \mu \mathrm{m}, P=0.47)$ and microcirculation densities $\left(22.1 \pm 2.8\right.$ vs. $22.5 \pm 1.5$ vessels $\left./ \mathrm{mm}^{2}, P=0.22\right)$.

Within individual animals, regional analysis between segments with and without MPDs demonstrated similar microvessel diameters $(7.8 \pm 1.8$ vs. $7.6 \pm 1.8 \mu \mathrm{m}, P=0.37)$ and microcirculation densities $\left(22.7 \pm 7.4\right.$ vs. $21.3 \pm 8.1$ vessels $\left./ \mathrm{mm}^{2}, P=0.3\right)$.

Mononuclear infiltrating cells were identified near macro- and microvessels in infected animals (Fig. 6), but this phenomenon was not exclusive to animals and segments with MPDs.

\section{DISCUSSION}

The present study demonstrated that in an experimental hamster model of CCC, resting MPDs are frequent and involve a significant extent of the LV myocardium. MPDs correlate with systolic LV dysfunction and exhibit a topographic correlation with changes in WMSi. Despite severity and occurrence at rest, MPD regions do not correlate topographically with significant transmural fibrosis or microvessel depletion. Rather, MPD regions typically correspond to metabolically viable myocardium with more extensive inflammation.

\section{The Hamster Model of CCC}

The experimental model used in our study reproduced the general histopathologic abnormalities classically described in this experimental model of CCC in hamsters, that is, diffuse myocarditis with mononuclear inflammatory infiltration associated with myocytolytic injury and mild interstitial and perivascular fibrosis (17).
Importantly, over the time frame chosen to investigate this model of CCC, we found significant heterogeneity in the individual degree of LV systolic function involvement and of histologic changes, consistent with previous reports (17). Accordingly, only $29 \%$ of the animals exhibited a significant reduction of LVEF over the course of the study.

This peculiarity of CCC in the hamster corresponds to the natural evolution of Chagas cardiomyopathy in humans, wherein only $20 \%-30 \%$ of chronically infected subjects develop late heart disease, with a variable degree of regional and global LV systolic dysfunction (24).

\section{Myocardial Perfusion Disorders}

In vivo high-resolution SPECT imaging revealed the presence of resting MPDs in 50\% of CCC animals. Regional analysis showed a topographic correlation between MPDs and abnormal wall motion, indicating pathophysiology rather than technical artifacts. In addition, we observed larger MPDs at 10 mo after infection than at $6 \mathrm{mo}$, suggesting that the myocardial perfusion derangement increases as the disease progresses.

Severe resting MPDs would be consistent with myocyte loss in chronic ischemia. However, histologic examination did not reveal coalescent fibrosis or differences between segments with and without MPDs. These results indicate that the presence of regional transmural fibrosis was not responsible for the observed MPDs. Preserved uptake of ${ }^{18} \mathrm{~F}$-FDG further supports metabolically viable myocardium within these MPD regions. However, uptake of ${ }^{18} \mathrm{~F}$-FDG by inflammatory infiltration in these regions with MPDs could also contribute to the preserved ${ }^{18} \mathrm{~F}$-FDG uptake, as seen in previous work $(25,26)$.

MPDs were associated with inflammation, wherein the MPD area positively correlated with the intensity of inflammatory infiltration $(r=0.51, P=0.002)$, and infected animals with MPDs exhibited a 


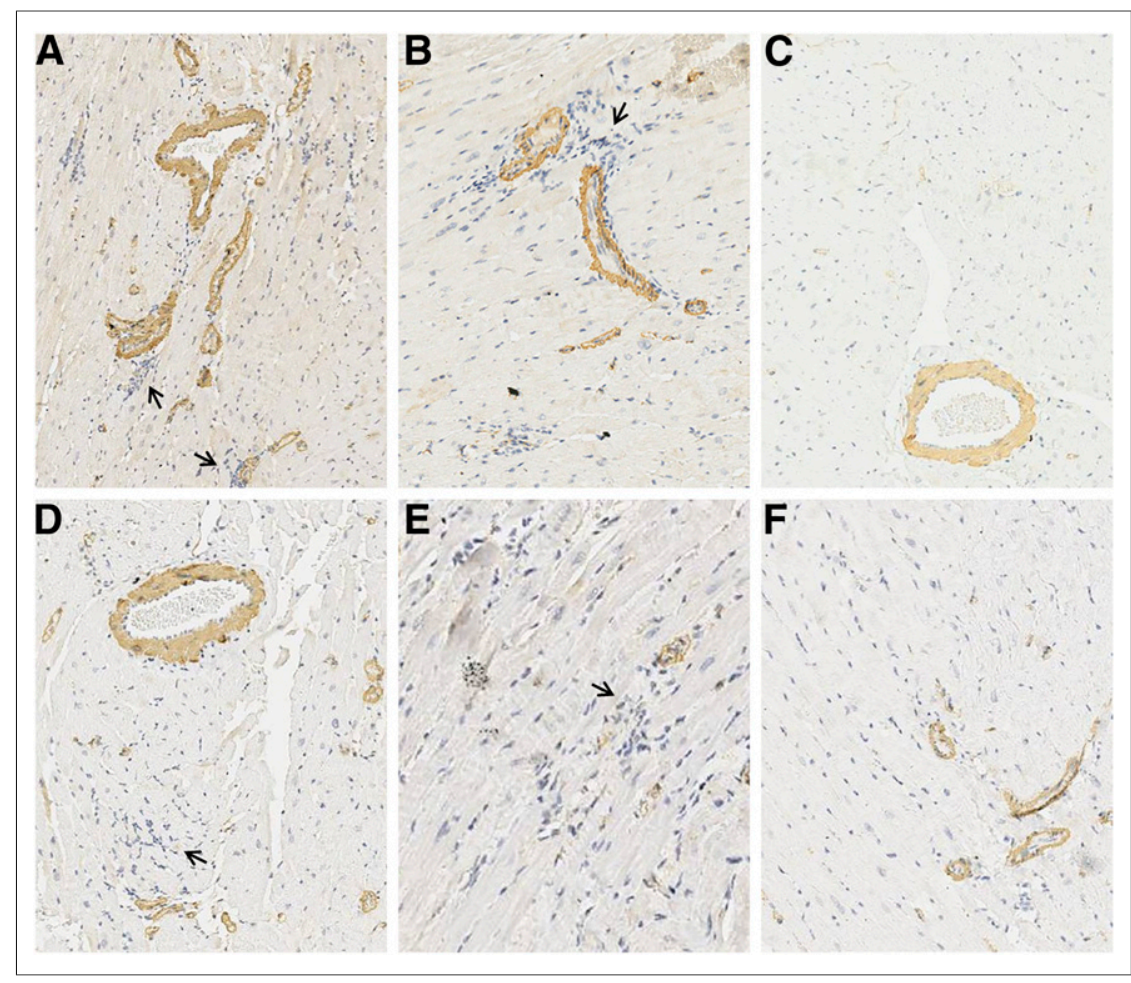

FIGURE 6. Immunohistochemistry sections showing mononuclear infiltrating cells (arrows) near macrocirculation $(A$ and $B$ ) and microcirculation $(D$ and $E)$. Similar changes were not observed in control animals $(\mathrm{C}$ and $\mathrm{F})$.

higher inflammation intensity. Taken together, these results indicate that MPDs are localized to regions of viable myocardium with reduction of perfusion secondary to inflammation.

Previous studies have demonstrated MPDs in active myocarditis of other origin. Sun et al. reported regions of myocardial hypoperfusion in $100 \%$ of patients with Coxsackie viral myocarditis, including 28\% with severe MDPs (27). Similarly, 15 of 20 patients with myocarditis due to systemic lupus erythematosus displayed reversible and fixed MDPs on ${ }^{99 \mathrm{~m}} \mathrm{Tc}$-sestamibi imaging (28).

To our knowledge, our study provides the first evidence that such a myocardial perfusion disturbance is present in association with low-grade diffuse, chronic Chagas myocarditis.

\section{Correlation Between Perfusion Defects and Systolic LV Function}

The present results show that infected animals with MPDs had lower LVEFs and greater derangement of WMSi. There was also a negative correlation between MPD extent and LVEF. These results indicate an association between myocardial perfusion and systolic LV dysfunction in CCC and corroborate the findings of clinical studies (7).

A previous clinical study also suggested that coronary microvascular ischemia may partly underlie the mechanism of myocardial dysfunction in CCC. In patients with reversible MPDs and normal coronary arteries on angiography, progression of reversible defects to fixed defects was associated with declining segmental and global systolic function over 5.6 y (12).

Abnormal myocardial perfusion and myocardial contractile dysfunction in CCC may derive from ischemic damage, similar to myocardial hibernation secondary to ischemia (4). Alternatively, perfusion and myocardial contractility abnormality may be caused by an underlying inflammatory response. As such, perfusion abnormalities may provide a surrogate marker of inflammation before LV contractile dysfunction and irreversible tissue injury.

\section{Topography of Myocardial Perfusion Disorders}

The MPDs observed in T. cruzi-infected animals were predominantly found in the apical LV region. Preferential regional involvement of the apical region has also been reported in scintigraphic studies of patients with $\operatorname{CCC}(7,12)$, although the mechanism is unknown.

The present results suggest involvement of inflammation as a mechanism leading to MPDs, since the animals with MPDs had higher inflammation than animals with normal perfusion.

In fact, autopsy observations in humans (29) and experimental studies in CCC $(14,30)$ have demonstrated structural and functional abnormalities of the coronary microcirculation that appear to be caused by the inflammatory infiltration into surrounding tissue $(8,31)$ and appear to be associated with the production of vasoactive substances $(32,33)$. These changes, including arteriolar spasm and intima proliferation leading to luminal obstruction (30), formation of occlusive platelet plugs (14), and vascular rarefaction (16), would lead to regional myocardial ischemia. However, in the present study, such changes were not observed, possibly reflecting a species difference in hamsters or in progression of disease in this model.

The absence of structural changes of the coronary microcirculation indirectly supports the alternative hypothesis that functional disorders in the control of myocardial blood flow may contribute to MPDs in CCC (34).

Another alternative mechanism may be mitochondrial dysfunction, which can lead to lower resting uptake of ${ }^{99 \mathrm{~m}} \mathrm{Tc}$-sestamibi by myocardium. It is known that CCC patients have high levels of interferon- $\gamma$ and tumor necrosis factor- $\alpha$. These cytokines, as well as $T$. cruzi, are associated with iNOS upregulation and morphologic and functional changes to mitochondria (35). Mitochondrial dysfunction alters uptake and washout of ${ }^{99 \mathrm{~m}} \mathrm{Tc}$-sestamibi, which accumulates on mitochondria $(36,37)$. Thus, defects in ${ }^{99 m} \mathrm{Tc}-$ sestamibi uptake relate to inflammation (and suggest mitochondrial dysfunction) without true hypoperfusion.

\section{Limitations}

Our assessment of myocardial perfusion using SPECT was semiquantitative, such that regions with MPDs might have corresponded to myocardial segments more severely hypoperfused than areas without MPDs. We cannot exclude the possibility that areas considered to be normal may show reduced myocardial blood flow if assessed with other, more quantitative, methods, such as ${ }^{13} \mathrm{~N}$ ammonia by PET imaging. Nevertheless, the regional heterogeneity observed on SPECT perfusion images indicated more significant hypoperfusion in the affected regions. Moreover, SPECT is the more widely available and routinely used modality, supporting clinical translation of the present findings. 


\section{Implications}

The present results support the hypothesis that the reduction of resting myocardial perfusion occurring in CCC involves LV areas with systolic dysfunction but metabolically viable myocardium. These changes are associated with inflammation and may be involved in tissue damage and the progression of contractile dysfunction in CCC. Myocardial inflammation has become a promising target in cardiovascular disease. These results provide the foundation for further studies addressed to detection and monitoring of myocardial inflammation and translation to clinical application.

Moreover, these severe resting MPDs were not due to extensive fibrosis, suggesting that therapeutic interventions targeted to inflammation or hypoperfusion may improve the outcomes in CCC.

The association of MPDs with inflammation also suggests that reversible myocardial perfusion disorders detected by scintigraphy in humans and occurring at rest $(7,9,11)$ may provide a surrogate marker for the presence of inflammation, with a potential role in monitoring and risk stratification in CCC (38).

\section{CONCLUSION}

Resting MPDs occur frequently in the experimental hamster model of CCC, correlate with global and regional LV systolic dysfunction, and are associated with myocardial inflammation in viable tissue. Taken together, these findings provide the foundation for future prospective studies to determine the prognostic value of SPECT-derived MPDs for late LV dysfunction and to monitor therapeutic interventions targeted to modulating myocardial inflammation or restoring normal perfusion.

\section{DISCLOSURE}

Luciano Fonseca Lemos de Oliveira and Marcus Vinicius Simões received grants 2015/25209-5 and 2011/03261-4, respectively, from the São Paulo Research Foundation (FAPESP). No other potential conflict of interest relevant to this article was reported.

\section{REFERENCES}

1. Cucunubá ZM, Okuwoga O, Basanez MG, Nouvellet P. Increased mortality attributed to Chagas disease: a systematic review and meta-analysis. Parasit Vectors. 2016;9:42.

2. Garcia MN, Murray KO, Hotez PJ, et al. Development of Chagas cardiac manifestations among Texas blood donors. Am J Cardiol. 2015;115:113-117.

3. Nunes MC, Dones W, Morillo CA, Encina JJ, Ribeiro AL; Council on Chagas Disease of the Interamerican Society of Cardiology. Chagas disease: an overview of clinical and epidemiological aspects. J Am Coll Cardiol. 2013;62:767-776.

4. Marin-Neto JA, Cunha-Neto E, Maciel BC, Simoes MV. Pathogenesis of chronic Chagas heart disease. Circulation. 2007;115:1109-1123.

5. Simões MV, Ayres EM, Santos JL, et al. Detection of myocardial ischemia in chronic Chagas disease patients with atypic precordial pain by exercise and Holter tests. Arq Bras Cardiol. 1993;60:315-319.

6. de Almeida-Filho OC, Maciel BC, Schmidt A, Pazin-Filho A, Marin-Neto JA. Minor segmental dyssynergy reflects extensive myocardial damage and global left ventricle dysfunction in chronic Chagas disease. J Am Soc Echocardiogr. 2002;15:610-616.

7. Simões MV, Pintya AO, Bromberg-Marin G, et al. Relation of regional sympathetic denervation and myocardial perfusion disturbance to wall motion impairment in Chagas' cardiomyopathy. Am J Cardiol. 2000;86:975-981.

8. Marin-Neto JA, Simões MV, Rassi A Junior. Pathogenesis of chronic Chagas cardiomyopathy: the role of coronary microvascular derangements. Rev Soc Bras Med Trop. 2013;46:536-541.

9. Hagar JM, Rahimtoola SH. Chagas' heart disease in the United States. $N$ Engl J Med. 1991;325:763-768.

10. Punukollu G, Gowda RM, Khan IA, Navarro VS, Vasavada BC. Clinical aspects of the Chagas' heart disease. Int J Cardiol. 2007;115:279-283.

11. Marin-Neto JA, Marzullo P, Marcassa C, et al. Myocardial perfusion abnormalities in chronic Chagas' disease as detected by thallium-201 scintigraphy. Am J Cardiol. 1992;69:780-784.
12. Hiss FC, Lascala TF, Maciel BC, Marin-Neto JA, Simoes MV. Changes in myocardial perfusion correlate with deterioration of left ventricular systolic function in chronic Chagas' cardiomyopathy. JACC Cardiovasc Imaging. 2009;2:164-172.

13. Torres CM. Arteriosclerosis of the fine arterial branches of the myocardium (Chagas' coronaritis) and focal myocytolysis in chronic Chagas' heart disease [in Portuguese]. Hospital (Rio J). 1958;54:597-610.

14. Rossi MA, Goncalves S, Ribeiro-dos-Santos R. Experimental Trypanosoma cruzi cardiomyopathy in $\mathrm{BALB} / \mathrm{c}$ mice: the potential role of intravascular platelet aggregation in its genesis. Am J Pathol. 1984;114:209-216.

15. Tanowitz HB, Kaul DK, Chen B, et al. Compromised microcirculation in acute murine Trypanosoma cruzi infection. J Parasitol. 1996;82:124-130.

16. Ferreira CS, Lopes ER, Chapadeiro E, de Oliveira Almeida H, de Souza WF, da Silva Neto IJ. Post-mortem coronary angiography in chronic Chagas carditis: anatomo-radiologic correlation. Arq Bras Cardiol. 1980;34:81-86.

17. Bilate AM, Salemi VM, Ramires FJ, et al. The Syrian hamster as a model for the dilated cardiomyopathy of Chagas' disease: a quantitative echocardiographical and histopathological analysis. Microbes Infect. 2003;5:1116-1124.

18. Umezawa ES, Nascimento MS, Kesper N Jr, et al. Immunoblot assay using excreted-secreted antigens of Trypanosoma cruzi in serodiagnosis of congenital, acute, and chronic Chagas' disease. J Clin Microbiol. 1996;34:2143-2147.

19. Oliveira LFL, Romano MM, de Carvalho EE, et al. Histopathological correlates of global and segmental left ventricular systolic dysfunction in experimental chronic Chagas cardiomyopathy. J Am Heart Assoc. 2016;5:e002786.

20. Oliveira LF, Mejia J, de Carvalho EE, et al. Myocardial infarction area quantification using high-resolution SPECT images in rats. Arq Bras Cardiol. 2013;101:59-67.

21. de Morais SB, Silva LE, Lataro RM, et al. Mesenchymal stem cells improve heart rate variability and baroreflex sensitivity in rats with chronic heart failure. Stem Cells Dev. 2015;24:2181-2192.

22. Thackeray JT, Bankstahl JP, Wang Y, Wollert KC, Bengel FM. Clinically relevant strategies for lowering cardiomyocyte glucose uptake for ${ }^{18} \mathrm{~F}-\mathrm{FDG}$ imaging of myocardial inflammation in mice. Eur J Nucl Med Mol Imaging. 2015; 42:771-780.

23. Tavares Monteiro JA, da Silva ES, Raghavan ML, Puech-Leao P, de Lourdes Higuchi M, Otoch JP. Histologic, histochemical, and biomechanical properties of fragments isolated from the anterior wall of abdominal aortic aneurysms. $J$ Vasc Surg. 2014;59:1393-1401.e1-2.

24. Dias E, Laranja FS, Miranda A, Nobrega G. Chagas' disease: a clinical, epidemiologic, and pathologic study. Circulation. 1956;14:1035-1060.

25. Prado CM, Fine EJ, Koba W, et al. Micro-positron emission tomography in the evaluation of Trypanosoma cruzi-induced heart disease: comparison with other modalities. Am J Trop Med Hyg. 2009;81:900-905.

26. Salimy MS, Parwani PJ, Mukai K, Pampaloni MH, Flavell RR. Abnormal ${ }^{18}$ F-FDG and ${ }^{82} \mathrm{Rb}$ PET findings in Chagas heart disease. Clin Nucl Med. 2017;42:e265-e268.

27. Sun Y, Ma P, Bax JJ, et al. ${ }^{99 \mathrm{~m} T c-M I B I}$ myocardial perfusion imaging in myocarditis. Nucl Med Commun. 2003;24:779-783.

28. Laganà $\mathrm{B}$, Schillaci $\mathrm{O}$, Tubani L, et al. Lupus carditis: evaluation with technetium99m MIBI myocardial SPECT and heart rate variability. Angiology. 1999;50:143-148.

29. Torres CM. Myocytolysis and fibrosis of the myocardium in Chagas' disease. Mem Inst Oswaldo Cruz. 1960;58:161-182.

30. Factor SM, Cho S, Wittner M, Tanowitz H. Abnormalities of the coronary microcirculation in acute murine Chagas' disease. Am J Trop Med Hyg. 1985;34:246-253.

31. Rossi MA, Tanowitz HB, Malvestio LM, et al. Coronary microvascular disease in chronic Chagas cardiomyopathy including an overview on history, pathology, and other proposed pathogenic mechanisms. PLoS Negl Trop Dis. 2010;4:e674.

32. Petkova SB, Huang H, Factor SM, et al. The role of endothelin in the pathogenesis of Chagas' disease. Int J Parasitol. 2001;31:499-511.

33. Ashton AW, Mukherjee S, Nagajyothi FN, et al. Thromboxane A2 is a key regulator of pathogenesis during Trypanosoma cruzi infection. J Exp Med. 2007;204:929-940.

34. Torres FW, Acquatella H, Condado JA, Dinsmore R, Palacios IF. Coronary vascular reactivity is abnormal in patients with Chagas' heart disease. Am Heart J. 1995;129:995-1001.

35. Ferreira LR, Frade AF, Baron MA, et al. Interferon-gamma and other inflammatory mediators in cardiomyocyte signaling during Chagas disease cardiomyopathy. World J Cardiol. 2014;6:782-790.

36. Hayashi $\mathrm{D}$, Ohshima $\mathrm{S}$, Isobe $\mathrm{S}$, et al. Increased ${ }^{99 \mathrm{~m}} \mathrm{Tc}$-sestamibi washout reflects impaired myocardial contractile and relaxation reserve during dobutamine stress due to mitochondrial dysfunction in dilated cardiomyopathy patients. J Am Coll Cardiol. 2013;61:2007-2017.

37. Masuda A, Yoshinaga K, Naya M, et al. Accelerated ${ }^{99 m}$ Tc-sestamibi clearance associated with mitochondrial dysfunction and regional left ventricular dysfunction in reperfused myocardium in patients with acute coronary syndrome. EJNMMI Res. 2016;6:41.

38. Schwartz RG, Wexler O. Early identification and monitoring progression of Chagas' cardiomyopathy with SPECT myocardial perfusion imaging. JACC Cardiovasc Imaging. 2009;2:173-175. 